Supporting Information

\title{
Using Silver Nanoparticles Embedded Silica Metafilms as Substrates to Enhance Performance of Perovskite Photodetectors
}

Bo Liu, ${ }^{*}{ }^{\dagger}$ Rithvik R. Gutha, ${ }^{\ddagger}$ Bhupal Kattel,${ }^{\dagger}$ Mohammed Alamri,,$^{\dagger}$ Maogang Gong, ${ }^{\dagger}$ Seyed M. Sadeghi, ${ }^{\ddagger}$ Wai-Lun Chan,$^{\dagger}$ and Judy Z. $W u^{*}{ }^{\dagger}$

$\dagger$ Department of Physics and Astronomy, The University of Kansas, Lawrence, Kansas 66045, USA

I Department of Physics and Astronomy, The University of Alabama in Huntsville, Huntsville, Alabama 35899, USA

* Corresponding authors: liubo@ku.edu; jwu@,ku.edu 


\section{A. a failed attempt of integrating metal NPs in perovskite/graphene photodetectors}

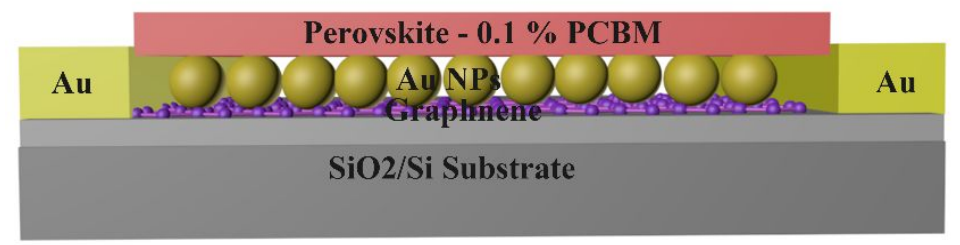

Figure S1. Perovskite-0.1\% PCBM/graphene photodetectors with Au NPs placed between graphene and perovskite layer.

The design of perovskite/graphene heterojunction photodetectors on Ag NPs-silica metafilm in this work was developed from a failed attempt with device structure shown in Figure S1. In the failed device, plasmonic Au NPs were placed between perovskite film and graphene. The perovskite film was mixed with $0.1 \%[6,6]-$ phenyl- $\mathrm{C}_{61}$-butyric acid methyl ester (PCBM), which serves to improve electron-hole separation efficiency by selective trapping of electrons, as demonstrated in the early works ${ }^{1-2}$.

a)

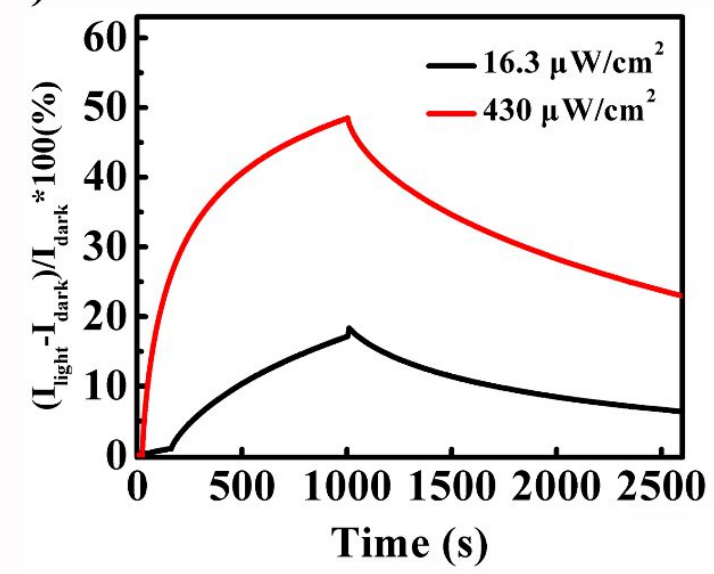

b)

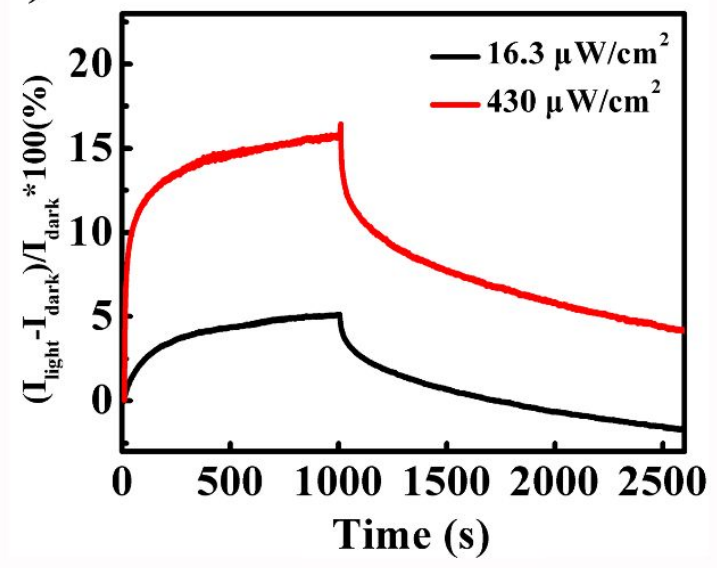

Figure S2. Photoresponse of perovskite - $0.1 \%$ PCBM/graphene photodetectors (a) without Au NPs and (b) with Au NPs placed between graphene and perovskite layer. Incident intensities were 16.3 $\mu \mathrm{W} / \mathrm{cm}^{2}$ (black) and $430 \mu \mathrm{W} / \mathrm{cm}^{2}$ (red), respectively.

Figure S2 compares the photoresponse of the Perovskite- $0.1 \%$ PCBM/graphene photodetectors without and with $\mathrm{Au}$ NPs placed between graphene and perovskite layer measured at incident intensities of $16.3 \mu \mathrm{W} / \mathrm{cm}^{2}$ and $430 \mu \mathrm{W} / \mathrm{cm}^{2}$. Obviously much faster response was achieved in the perovskite photodetector with integration of Au NPs $(68 \mathrm{~s}$ compared to $291 \mathrm{~s}$ ). This could be attributed to the extra absorption occurs at the region close to graphene in the latter device from LSPR enhanced EM field makes the photon-electron excitation saturate more rapidly. However, contrary to the purpose we applied Au NPs into the photodetector, photoresponse in the device with Au NPs was reduced by $2 / 3$ compared with the 
counterpart without Au NPs. The reason was assumed as the direct contact of Au NPs and perovskite without a separating layer causes both energy and charge loss, thus neutralized the field enhancement from Au NPs plasmonic effect. Also, non-negligible heat transferring from $\mathrm{Au}$ NPs to perovskite could decline the performance of photodetector especially sensitized by perovskite owing to its poor thermal stability. This result indicates the importance of NPs-silica metafilm when integrating plasmonic metal NPs in perovskite photodetectors.

B. Figure-of-Merit Detectivity $\left(D^{*}\right)$ and Photoconductive Gain $(G)$ of the perovskite/graphene photodetectors with and without Ag NPs-silica metafilm

The figure-of-merit detectivity $\left(\boldsymbol{D}^{*}\right)$ is expressed as the following formula

$$
D^{*}=\frac{R \times A^{\frac{1}{2}}}{\frac{1}{i_{n}^{2} \frac{1}{2}}} \text { (jones) }
$$

where $R$ is the photoresponsivity, A is the detection area with unit of $\mathrm{cm}^{2}$, and $\overline{i_{n}{ }^{2}}$ is mean square noise current calculated from noise power density. Noise power density of both devices with and without Ag NPs-silica metafilm are given in Figure S3a, and incident power dependent $D^{*}$ are plotted in Figure $\mathbf{S 3 b}$.
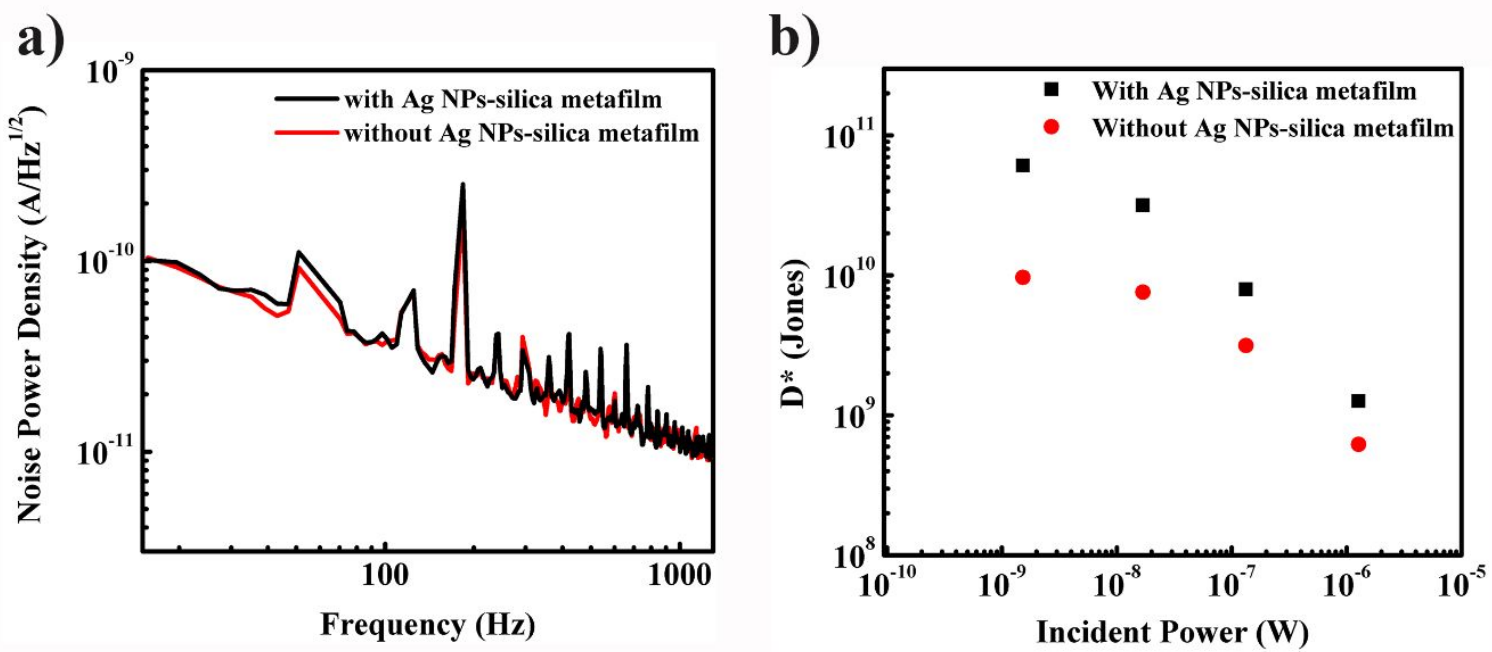

Figure S3. (a) Noise power density and (b) $D^{*}$ vs. incident power of perovskite photodetectors with (black) and without (red) Ag NPs-silica metafilm. $D^{*}$ were calculated using $R$ values measured under $585 \mathrm{~nm}$ illumination and Bias voltage of $0.1 \mathrm{~V}$

In this work, perovskite/graphene photodetectors with and without Ag NPs-silica metafilm all have the same detection area. Also, all the devices are fabricated on the substrate and share the same graphene, thus the mean square noise currents for both devices are almost identical 
and were calculated to be $4.3 \times 10^{-10} \mathrm{~A} / \mathrm{Hz}^{1 / 2}$ and $4.01 \times 10^{-10} \mathrm{~A} / \mathrm{Hz}^{1 / 2}$, respectively. Therefore, it is not surprising that the incident power dependent detectivity (Figure S3b) of both devices shows the same trend as incident power dependent responsivity (Figure 6d), as $R$ is proportional to $D^{*}$ from the definition.

The photoconductive gain $(\boldsymbol{G})$ is determined by the quotient of charge carrier lifetime $(\tau)$ and the transit time $\left(t_{\text {Transit }}\right)$ in the graphene channel:

$$
\mathrm{G}=\frac{\tau}{t_{\text {Transit }}}=\frac{\tau}{L^{2} \mu^{-1} V_{s d}{ }^{-1}}
$$

where $L$ is the graphene channel length, $\mu$ is the charge carrier mobility, and $V_{s d}$ is the source-drain bias voltage. In our case, $L=1 \mathrm{~mm}, V_{s d}=0.1 \mathrm{~V}$, and $\mu \sim 53.6 \mathrm{~cm}^{2} /(\mathrm{V} \mathrm{s})$. Charge carrier lifetime is obtained by fitting in the Figure $6 \mathrm{~b}$ where we get $\tau=2.74 \mathrm{~s}$ for the device with Ag NPs-silica metafilm and $\tau=2.52 \mathrm{~s}$ for the device without Ag NPs-silica metafilm. Therefore, $G$ is calculated to be $1.47 \times 10^{3}$ and $1.35 \times 10^{3}$ for the device with and without $\mathrm{Ag}$ NPs-silica metafilm, respectively.

\section{References}

1. Qin, L.; Wu, L. P.; Kattel, B.; Li, C. H.; Zhang, Y.; Hou, Y. B.; Wu, J.; Chan, W. L., Using Bulk Heterojunctions and Selective Electron Trapping to Enhance the Responsivity of Perovskite-Graphene Photodetectors. Adv. Funct. Mater. 2017, 27 (47), 1704173.

2. Wu, L.; Qin, L.; Zhang, Y.; Alamri, M.; Gong, M.; Zhang, W.; Zhang, D.; Chan, W. L.; $\mathrm{Wu}$, J. Z., High-Sensitivity Light Detection Via Gate Tuning of Organometallic Perovskite/PCBM Bulk Heterojunctions on Ferroelectric $\mathrm{Pb}_{0.92} \mathrm{La}_{0.08} \mathrm{Zr}_{0.52} \mathrm{Ti}_{0.48} \mathrm{O}_{3}$ Gated Graphene Field Effect Transistors. ACS Appl. Mater. Interfaces 2018, 10 (15), 12824-12830. 